\title{
Distribution function of the blow up time of the solution of an anticipating random fatigue equation
}

\author{
Liliana Peralta*
}

September 23, 2019

\begin{abstract}
In this paper, we study the distribution function of the time of explosion of a stochastic differential equation modeling the length of the dominant crack due to fatigue. The main novelty is that initial condition is regarded as an anticipating random variable and the stochastic integral is in the forward sense.

Under suitable conditions, we use the substitution formula from Russo and Vallois to find the local solution of this equation. Then, we find the law of blow up time by proving some results on barrier crossing probabilities of Brownian bridge.
\end{abstract}

Keywords: Stochastic differential equations, random fatigue, explosion time, forward integral, Brownian bridge.

MSC2010: 60H10, 91B70, 60H05, 60J65

\section{Introduction}

Nowadays, most of the components of certain types of structures present discontinuities or notches, which usually appear by the manufacturing process and operating conditions. With time and due to cyclic loads, these discontinuities cause the appearance of cracks, and in turn generate high concentration of efforts that could produce failures in the structures, whence, the rate of propagation of a crack varies in time.

Over time, different models have been developed to predict the propagation of cracks, relating the properties of the material with geometric characteristics and different loading conditions. In 1963 the well known Paris' law [32] of fracture mechanics is introduced and, since then, several generalizations of this model have been made. As experiments have shown, the randomness is a characteristic feature of crack growth and stochastic components have been incorporated to the models to take into account the typical variability of the dynamics of cracks on solids.

The following stochastic model

$$
d L_{t}=\left(c_{1} L_{s}^{p}+\frac{p c_{2}^{2}}{2} L_{s}^{2 p-1}\right) d t+\int_{0}^{t} c_{2} L_{s}^{p} d W_{t}, \quad L_{0}=l_{0}
$$

is a generalization of the classical Paris' law and it was proposed by Sobczyk ([37], [38]) to represent the time evolution of the length of the dominant crack. Here $W$ is a Brownian motion defined in a filtered probability space $\left(\Omega, \mathcal{F},\left\{\mathcal{F}_{t}\right\}_{t \geq 0}, P\right)$ which filtration satisfies the usual conditions, while $c_{1}$ and $c_{2}$ are positive constants depending on various parameters used to describe the effects of random loading on crack growth, in particular the intensity factor range and ratio of the applied stress. The variable $p$ is a positive constant and it is determined from experimental data (see, for instance [13], [40]). When $p=1$, the coefficients of equation (1) have linear growth and thus its solution is global, i.e., it is well defined for all $t \geq 0$. Nevertheless, it has been found that several materials have values of $p$ greater than one (see [27]), varying

* Centro de Investigación en Matemáticas, UAEH, Carretera Pachuca-Tulancingo km 4.5 Pachuca, Hidalgo 42184, Mexico E-mail: liliana_peralta@uaeh.edu.mx 
according to the type of material, the environmental conditions and the type of load applied. In this case, the solution $L$ may explode in finite time (see [4], [26]) which, at the mechanical level, translates into the precise instant where the fracture occurs.

The point of the fracture is a relevant topic and has attracted attention in the recent past due to its numerous applications. For instance in [12], the authors investigate the mechanisms of fatigue crack growth in polycarbonate polyurethane, a material widely used in orthopedic applications due to its cartilage-like, hygroscopic, and elastomeric properties. Other interesting applications can be found in the fields of mechanics, aviation, shipping. We refer the interested reader to the works [5], [8], [24].

In the present paper, we propose to find the distribution of the blow up time of the solution of process (1) when $p>1$ but, different from [37] and other related works, we replace the deterministic initial condition $l_{0}$ by a more general anticipating random variable $I$. As a consequence, it will be necessary to use tools of anticipating stochastic calculus (see, for instance [18], [29], [31]) in order to achieve the goal of the work. The anticipating stochastic calculus allows the study of stochastic differential equations (SDE) where the coefficients or the initial condition may depend on future information.

The stochastic integral in equation (1) is understood in the classical Itô sense (see, e.g. [14]) and is defined for adapted processes to the information generated by the Brownian motion $W$, but in this proposal, taking into account the new characteristic of the initial condition, we need to use an integral which allows the integration of non-adapted processes. The anticipating stochastic integral known as forward, defined by Russo and Vallois in [35], is an extension of the well known Itô's integral for the case of anticipating integrands and coincides with the Itô's one when the integrands are square integrable, measurable and $\mathcal{F}_{t}$-adapted processes.

To achieve the objective of this paper, first in Section 2, we will be prove using the substitution formula for the forward integral [35], that the local solution of the anticipating stochastic equation is given as follows:

Theorem 1.1. Let $\bar{t}>0$ and $I>0$ a random variable. Then the process

$$
\left((I)^{1-p}-c_{1}(p-1) .-c_{2}(p-1) W .\right)^{\frac{p}{1-p}}
$$

belongs to Dom $\delta_{\bar{t}, l o c}^{-}$(see Definition 2.4) on the set

$$
A_{\bar{t}}=\left\{(I)^{1-p}-c_{1}(p-1) \bar{t}-c_{2}(p-1) \sup _{0 \leq s \leq \bar{t}} W_{s}>0\right\}
$$

for any $t \in(0, \bar{t}]$. Furthermore, for $t \leq \bar{t}, p>1$, and $I>0$ we have that

$$
L_{t}^{I}=I+\int_{0}^{t}\left(c_{1}\left(L_{s}^{I}\right)^{p}+\frac{p c_{2}^{2}}{2}\left(L_{s}^{I}\right)^{2 p-1}\right) d s+\int_{0}^{t} c_{2}\left(L_{s}^{I}\right)^{p} d^{-} W_{s}
$$

is well defined on $A_{\bar{t}}$, where

$$
L_{t}^{x}=\left(\left(\frac{1}{x}\right)^{p-1}-c_{1}(p-1) t-c_{2}(p-1) W_{t}\right)^{\frac{1}{1-p}}, \quad t \geq 0, x \in \mathbb{R}
$$

Since we are considering the case $p>1$, notice that (3) might have paths that reach infinite values in finite times. The first instant in which this phenomenon occurs is called the time of the explosion of the process and hereinafter we will denote it by $\tau$.

In Section 3, when the initial condition for (2) is given by $I=g\left(W_{T}\right)$ for a borel function $g$ : $\mathbb{R} \rightarrow(0, \infty)$, we will compute the probability distribution of the time of explosion of the solution of process (2). To find this distribution, we will use results on barrier crossing probabilities of Brownian bridge (see e. g. [3],[34]), namely, we will prove conditional crossing-probability for a linear boundary of the form

$$
P\left(\bigcup_{0 \leq t \leq r}\left\{W_{t} \geq a-b t\right\} \mid W_{T}=x\right) .
$$


The barrier crossing probabilities that we will prove are a generalization of those found in [6] and they have been extended for the case of piecewise-linear boundary in [1]. For the sake of conciseness, we will present proofs that are useful and adapted for our purposes. Since the moment of the fracture may occur before or after the time $T$ we pay attention to the cases $r \leq T$ and $r>T$ in the probability (4).

With all of the above, we find that the distribution function of the explosion time of the solution of (2) is:

Theorem 1.2. Let $\Phi$ be the standard normal distribution function, $\phi\left(0, \sigma^{2}\right)$ be the normal density function with mean zero and variance $\sigma^{2}$ and $\tau$ be the blow up time of solution of the anticipating process (2) given by

$$
\tau=\inf \left\{t>0: W_{t} \geq \frac{1}{c_{2}(p-1) I^{p-1}}-\frac{c_{1}}{c_{2}} t\right\} .
$$

Define

$$
a(x)=\frac{1}{c_{2}(p-1)(g(x))^{p-1}}-x-\frac{c_{1} T}{c_{2}} \text { and } v=\frac{1}{r}-\frac{1}{T} .
$$

Then

i) If $r<T$,

$$
\begin{aligned}
& P(\tau \leq r) \\
& \quad=1-\int_{-\infty}^{\infty} \Phi\left(\frac{a(x)}{T \sqrt{v}}+\frac{\sqrt{v}}{c_{2}(p-1)(g(x))^{p-1}}\right) \phi(0, T) d x \\
& \quad+\int_{-\infty}^{\infty} \exp \left(\frac{-2 a(x)}{T c_{2}(p-1)(g(x))^{p-1}}\right) \Phi\left(\frac{a(x)}{T \sqrt{v}}-\frac{\sqrt{v}}{c_{2}(p-1)(g(x))^{p-1}}\right) \phi(0, T) d x .
\end{aligned}
$$

ii) If $r=T$,

$$
\begin{aligned}
P(\tau \leq r)= & -\int_{-\infty}^{\infty} I_{\{a(x)>0\}} \phi(0, T) d x \\
& +\int_{-\infty}^{\infty} \exp \left(\frac{-2 a(x)}{T c_{2}(p-1)(g(x))^{p-1}}\right) I_{\{a(x)>0\}} \phi(0, T) d x .
\end{aligned}
$$

iii) If $r>T$,

$$
\begin{aligned}
& P(\tau \leq r) \\
& =\int_{-\infty}^{\infty} \exp \left(\frac{-2 a(x)}{T c_{2}(p-1)(g(x))^{p-1}}\right) \Phi\left(\frac{a(x)}{\sqrt{r-T}}-\frac{c_{1} \sqrt{r-T}}{c_{2}}\right) I_{\{a(x)>0\}} \phi(0, T) d x \\
& \quad-\int_{-\infty}^{\infty} \exp \left(\frac{-2 a(x)}{T c_{2}(p-1)(g(x))^{p-1}}+\frac{2 a(x) c_{1}}{c_{2}}\right) \\
& \quad \times \Phi\left(\frac{-a(x)}{\sqrt{r-T}}-\frac{c_{1} \sqrt{r-T}}{c_{2}}\right) I_{\{a(x)>0\}} \phi(0, T) d x \\
& +1-\int_{-\infty}^{\infty} \Phi\left(\frac{a(x)}{\sqrt{r-T}}-\frac{c_{1} \sqrt{r-T}}{c_{2}}\right) I_{\{a(x)>0\}} \phi(0, T) d x \\
& \quad+\int_{-\infty}^{\infty} \exp \left(\frac{2 a(x) c_{1}}{c_{2}}\right) \Phi\left(\frac{-a(x)}{\sqrt{r-T}}-\frac{c_{1} \sqrt{r-T}}{c_{2}}\right) I_{\{a(x)>0\}} \phi(0, T) d x .
\end{aligned}
$$

\section{The anticipating case}

In this section we find the solution of equation (2) in which the involved stochastic process

$$
\left\{\int_{0}^{t} c_{2}\left(L_{s}^{I}\right)^{p} d^{-} W_{s},\right\}_{t \geq 0}
$$


is a forward integral. The forward integral was introduced by Russo and Vallois in their seminal work [35] and is an extension of Itô's one. We present its definition.

Definition 2.1. Let $T>0$ and $v$ be a measurable process with integrable trajectories. We say that $v$ is forward integrable (i.e. $v \in$ Dom $\delta_{T}^{-}$) if

$$
\frac{1}{\epsilon} \int_{0}^{T} v_{s}\left(W_{(s+\epsilon) \wedge T}-W_{s}\right) d s
$$

converges in probability as $\epsilon \downarrow 0$. We denote this limit by $\int_{0}^{T} v_{s} d_{s}^{-} W_{s}$.

In order to achieve our goal, we will use the so-called substitution formula for the forward integral (see, for instance, [28], [35]), which we enunciate below.

To this end let $\mathcal{R}$ be the set of random fields $X=\left\{X_{t}(u): t \in[0, T], u \in \mathbb{R}\right\}$ which are $\mathcal{P} \otimes \mathcal{B}(\mathbb{R})$-measurables, where $\mathcal{P}$ is the $\sigma$-algebra generated by the previsible processes. We have the following:

Theorem 2.2. Consider the class of processes

$$
\begin{aligned}
\mathcal{R}_{2}= & \left\{X \in \mathcal{R}: X(0) \in L^{2}([0, T]), \quad X_{t}(u)\right. \text { is differentiable in } \\
& \left.u \text { and } \int_{-n}^{n} \int_{0}^{T} X_{t}^{\prime}(u)^{2} d t d u<\infty \quad \forall n \in \mathbb{N}\right\} .
\end{aligned}
$$

If $X \in \mathcal{R}_{2}$, then for every random variable $Z, X(Z) \in D o m \delta_{T}^{-}$and

$$
\int_{0}^{T} X_{s}(Z) d^{-} W_{s}=\left(\int_{0}^{T} X_{s}(u) d W_{s}\right)_{u=Z} .
$$

Bearing in mind the above statements we shall look for the solution of process (1) by using the substitution formula. First, to fix ideas we set $L_{0}=x$, for $x \in \mathbb{R}$. Transforming the process (1) to a more convenient one, i.e., $\left\{\left(L_{t}\right)^{1-p}\right\}_{t \geq 0}$ and applying Itô's formula, we find that the solution is given by

$$
L_{t}^{x}=\left(\left(\frac{1}{x}\right)^{p-1}-c_{1}(p-1) t-c_{2}(p-1) W_{t}\right)^{\frac{1}{1-p}}, \quad t \geq 0 .
$$

Since $p>1$, the process (6) may explode with positive probability (see [26]) and therefore it is necessary to use that the forward integral satisfies the following local property which was proved in [28].

Lemma 2.3. Let $v, u \in$ Dom $\delta_{T}^{-}$be mesurable processes and $A \in \mathcal{F}$ such that

$$
u_{t}=v_{t} \quad \text { a.s. on } A \times[0, T] .
$$

Then $\int_{0}^{T} v_{s} d_{s}^{-} W_{s}=\int_{0}^{T} u_{s} d_{s}^{-} W_{s}$ a.s. on $A$.

As a consequence of Lemma 2.3, the domain of forward integral can be extended in the following manner.

Definition 2.4. A process $v$ is locally forward integrable (i.e. $v \in$ Dom $\delta_{T, l o c}^{-}$) on a measurable set $A \in \mathcal{F}$, if there exist a subsequence $\left\{\left(v^{n}, A_{n}\right)\right\}_{n \in \mathbb{N}}$ in Dom $\delta_{T}^{-} \times \mathcal{F}$, such that:

i) $A_{n} \nearrow A$ a.s.

ii) $v=v^{n}$ a.s. on $A_{n} \times[0, T]$.

Thus,

$$
\int_{0}^{T} v_{s} d^{-} W_{s} \equiv \int_{0}^{T} v_{s}^{n} d^{-} W_{s} \quad \text { on } \quad A_{n} .
$$

Remark 2.5. Lemma 2.3 implies that Definition 2.4 is independent of the localizing sequence $\left\{\left(v^{n}, A_{n}\right)\right\}_{n \in \mathbb{N}}$. 
Before we prove the main result of this section, we shall need to define the following functions. For $m \in \mathbb{N}$ large enough, let $\varphi_{m} \in C^{\infty}(\mathbb{R})$ be bounded functions satisfying

$$
\varphi_{m}(x)=\left\{\begin{array}{cl}
\frac{2}{m^{(p-1) / p}}, & x \leq \frac{2}{m^{(p-1) / p}} \\
x, & x \in\left(\frac{3}{m^{(p-1) / p}}, m\right) \\
m+1, & x \geq m+1
\end{array}\right.
$$

The existence of these functions can be verified on [20]. It is clear that $\varphi_{m}(x)>0$ for all $x \in \mathbb{R}$. Hence, for $t>0$, the Itô's stochastic integrals

$$
\int_{0}^{t} c_{2}\left(\varphi_{m}\left(\left(\frac{1}{x}\right)^{p-1}-c_{1}(p-1) s-c_{2}(p-1) W_{s}\right)\right)^{\frac{p}{1-p}} d W_{s}
$$

are well-defined for any $t>0$. In addition, it is easy to see that the random fields

$$
\left(\varphi_{m}\left(u^{p-1}-c_{1}(p-1) s-c_{2}(p-1) W_{s}\right)\right)^{\frac{p}{1-p}},
$$

satisfy the hypotheses of Theorem 2.2 and therefore the forward integrals

$$
\int_{0}^{t} c_{2}\left(\varphi_{m}\left((I)^{1-p}-c_{1}(p-1) s-c_{2}(p-1) W_{s}\right)\right)^{\frac{p}{1-p}} d^{-} W_{s}
$$

are well-defined too.

Now we are in position to present the main result of this section.

Proof of the Theorem 1.1. For $m \in \mathbb{N}$ large enough, we consider the sets

$$
\begin{aligned}
A_{m}= & \left\{(I)^{1-p}-c_{1}(1-p) s-c_{2}(p-1) \sup _{0 \leq s \leq \bar{t}} W_{s} \geq \frac{3}{m^{(p-1) / p}},\right. \\
& \left.(I)^{1-p}-m \leq c_{2}(p-1) \inf _{0 \leq s \leq \bar{t}} W_{s}\right\} .
\end{aligned}
$$

Accordingly with the definition of functions (7), we can verify that

$$
\varphi_{m}\left((I)^{1-p}-c_{1}(p-1) s-c_{2}(p-1) W_{s}\right)=(I)^{1-p}-c_{1}(p-1) s-c_{2}(p-1) W_{s}
$$

on $A_{m} \times[0, t]$ a.s., for $t \leq \bar{t}$. Thus, from the substitution formula (5) in Theorem 2.2 we get

$$
\begin{aligned}
& 1_{A_{m}} \int_{0}^{t} c_{2}\left(\varphi_{m}\left((I)^{1-p}-c_{1}(p-1) s-c_{2}(p-1) W_{s}\right)\right)^{\frac{p}{1-p}} d^{-} W_{s} \\
& =\left(1_{\left\{x^{p-1}-c_{1}(p-1) s-c_{2}(p-1) \sup _{0 \leq s \leq \bar{t}} W_{s} \geq \frac{3}{m^{(p-1) / p}}, x^{p-1}-m \leq c_{2}(p-1) \inf _{0 \leq s \leq \bar{t}} W_{s}, \frac{1}{x}>0\right\}}\right. \\
& \left.\times \int_{0}^{t} c_{2}\left(\varphi_{m}\left(x^{p-1}-c_{1}(p-1) s-c_{2}(p-1) W_{s}\right)\right)^{\frac{p}{1-p}} d W_{s}\right)_{x=1 / I}
\end{aligned}
$$

and from equality (8) we have

$$
\begin{gathered}
=\left(1_{\left\{x^{p-1}-c_{1}(p-1) s-c_{2}(p-1) \sup _{0 \leq s \leq \bar{t}} W_{s} \geq \frac{3}{m^{(p-1) / p}}, x^{p-1}-m \leq c_{2}(p-1) \inf _{0 \leq s \leq \bar{t}} W_{s}, \frac{1}{x}>0\right\}}\right. \\
\left.\times \int_{0}^{t} c_{2}\left(x^{p-1}-c_{1}(p-1) s-c_{2}(p-1) W_{s}\right)^{\frac{p}{1-p}} d W_{s}\right)_{x=1 / I} .
\end{gathered}
$$

Replacing the integrand in the above equation by the formula defined in (6), it is obtained that

$$
\begin{aligned}
& 1_{A_{m}} \int_{0}^{t} c_{2}\left(\varphi_{m}\left((I)^{1-p}-c_{1}(p-1) s-c_{2}(p-1) W_{s}\right)\right)^{\frac{p}{1-p}} d^{-} W_{s}
\end{aligned}
$$

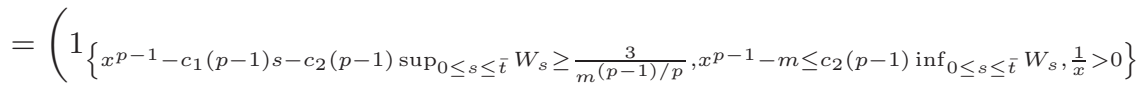




$$
\begin{gathered}
\left.\times \int_{0}^{t} c_{2}\left(L_{s}^{\frac{1}{x}}\right)^{p} d W_{s}\right)_{x=1 / I} \\
=\left(1_{\left\{x^{p-1}-c_{1}(p-1) s-c_{2}(p-1) \sup _{0 \leq s \leq \bar{t}} W_{s} \geq \frac{3}{\left.m^{(p-1) / p}, x^{p-1}-m \leq c_{2}(p-1) \inf _{0 \leq s \leq t} W_{s}, \frac{1}{x}>0\right\}}\right.}\right. \\
\left.\times\left\{L_{t}^{\frac{1}{x}}-\frac{1}{x}-\int_{0}^{t}\left(c_{1}\left(L_{s}^{\frac{1}{x}}\right)^{p}+\frac{p c_{2}^{2}}{2}\left(L_{s}^{\frac{1}{x}}\right)^{2 p-1}\right) d s\right\}\right)_{x=1 / I} \\
=1_{A_{m}}\left(L_{t}^{I}-I-\int_{0}^{t}\left(c_{1}\left(L_{s}^{I}\right)^{p}+\frac{p c_{2}^{2}}{2}\left(L_{s}^{I}\right)^{2 p-1}\right) d s\right),
\end{gathered}
$$

where the last equality follows from Fubini's Theorem.

Finally, since $A_{m} \nearrow A_{\bar{t}}$ a.s., then $\left\{\varphi_{m}\left(\left(\frac{1}{I}\right)^{p-1}-c_{1}(p-1) s-c_{2}(p-1) W_{s}\right), A_{m}\right\}$ is a localizing sequence for the process $\left(\frac{1}{I}\right)^{p-1}-c_{1}(p-1) .-c_{2}(p-1) W$. on $A_{\bar{t}}$. This concludes the proof.

\section{Life Distribution}

We have proved in Theorem 1.1 that the solution of the process (2) is given by

$$
L_{t}^{I}=\left((I)^{1-p}-c_{1}(p-1) t-c_{2}(p-1) W_{t}\right)^{\frac{1}{1-p}}, \quad t \geq 0
$$

and, as discussed before, we consider the random variable

$$
\tau=\inf \left\{t>0: W_{t} \geq \frac{1}{c_{2}(p-1) I^{p-1}}-\frac{c_{1}}{c_{2}} t\right\}
$$

as its blow up time.

To compute the distribution function of (9), we begin this section by proving crossing results for the Brownian bridge under conditions that will be sufficient for our main purpose. The proofs of this results follow a combination of invariance properties of standard Brownian motion in a similar fashion as [36].

We begin by recalling the following results (see, for instance [7], [10], [36]).

Proposition 3.1. Let $W$ be an standard Brownian motion and let $g(t)=a+b t$.

i) If $a, b>0$, then

$$
P\left(\bigcup_{0 \leq t}\left\{W_{t} \geq g(t)\right\}\right)=\exp (-2 a b) .
$$

If either $a \leq 0$ or $b \leq 0$, then the probability is 1 .

ii) If $a>0$ and $r<\infty$, then

$$
P\left(\bigcup_{0 \leq t \leq r}\left\{W_{t} \geq g(t)\right\}\right)=1-\Phi\left(\frac{a}{\sqrt{r}}+b \sqrt{r}\right)+\exp (-2 a b) \Phi\left(b \sqrt{r}-\frac{a}{\sqrt{r}}\right) .
$$

If $a \leq 0$, then the probability is $1 .{ }^{1}$

Now, we are ready to prove some crossing results concerning the Brownian bridge. The first result is the following.

Theorem 3.2. Let $\Phi$ be the normal standard distribution, $a, b, x \in \mathbb{R}, T, r>0$ and define $v=\frac{1}{r}-\frac{1}{T}$.

\footnotetext{
${ }^{1}$ In the original paper [36] there is a mismatched sign in the last term of (10). This was a typo that was later corrected in [1].
} 
a) If $a>0$ and $a-b T>x$, then

$$
P\left(\bigcup_{0 \leq t \leq T}\left\{W_{t} \geq a-b t\right\} \mid W_{T}=x\right)=\exp (-2 a(a-x-b T) / T) .
$$

If either $a \leq 0$ or $a-b T \leq x$, then the probability (11) is 1 .

b) Let $r<T$. If $a>0$, then

$$
\begin{aligned}
P\left(\bigcup_{0 \leq t \leq r}\left\{W_{t} \geq a-b t\right\} \mid W_{T}=x\right) \\
=1-\Phi\left(\frac{a-x-b T}{T \sqrt{v}}+a \sqrt{v}\right) \\
\quad+\exp (-2 a(a-x-b T) / T) \Phi\left(\frac{a-x-b T}{T \sqrt{v}}-a \sqrt{v}\right) .
\end{aligned}
$$

If $a \leq 0$, then the probability in (12) is 1 .

Proof. Assume that $r \leq T$. Using invariance properties of Brownian motion we get,

$$
\begin{aligned}
P\left(\bigcup_{0 \leq t \leq r}\left\{W_{t} \geq a-b t\right\} \mid W_{T}=x\right) & =P\left(\bigcup_{0 \leq t \leq r}\left\{t W_{\frac{1}{t}} \geq a-b t\right\} \mid T W_{\frac{1}{T}}=x\right) \\
& =P\left(\bigcup_{\frac{1}{r} \leq t}\left\{W_{t} \geq a t-b\right\} \mid W_{\frac{1}{T}}=\frac{x}{T}\right) .
\end{aligned}
$$

Since $r \leq T$ it is satisfied that $\frac{1}{r}=\frac{1}{T}+v$ for some $v \geq 0$, therefore we can rewrite the last expression in (13) as

$$
\begin{aligned}
& P\left(\bigcup_{\frac{1}{r} \leq t}\left\{W_{t} \geq a t-b\right\} \mid W_{\frac{1}{T}}=\frac{x}{T}\right) \\
& =P\left(\bigcup_{v \leq t-\frac{1}{T}}\left\{W_{\left(t-\frac{1}{T}\right)+\frac{1}{T}}-W_{\frac{1}{T}} \geq a\left(t-\frac{1}{T}\right)+\frac{a}{T}-b-\frac{x}{T}\right\} \mid W_{\frac{1}{T}}=\frac{x}{T}\right) \\
& =P\left(\bigcup_{v \leq t}\left\{W_{t+\frac{1}{T}}-W_{\frac{1}{T}} \geq a t+\frac{a}{T}-b-\frac{x}{T}\right\} \mid W_{\frac{1}{T}}=\frac{x}{T}\right) .
\end{aligned}
$$

Using the independence of increments of brownian motion we conclude

$$
P\left(\bigcup_{0 \leq t \leq r}\left\{W_{t} \geq a-b t\right\} \mid W_{T}=x\right)=P\left(\bigcup_{v \leq t}\left\{W_{t} \geq a t+\frac{a}{T}-b-\frac{x}{T}\right\}\right) .
$$

Observe that $r=T$ implies that $v=0$, therefore we can apply case i) of Proposition 3.1 to the right-hand side of equation (14) and thus a) follows immediately.

Now, in equality (14), let as assume that $v>0$, i.e., $r<T$ and, to simplify notation, we set $\tilde{b}=\frac{a}{T}-b-\frac{x}{T}$. Conditioning on the value of random variable $W_{v}$ and using once again invariance properties and independence of increments of brownian motion, we obtain

$$
\begin{aligned}
& P\left(\bigcup_{v \leq t}\left\{W_{t} \geq a t+\tilde{b}\right\}\right) \\
& \quad=\int_{-\infty}^{\infty} P\left(\bigcup_{v \leq t}\left\{W_{t} \geq a t+\tilde{b}\right\} \mid W_{v}=y\right) P\left(W_{v} \in d y\right)
\end{aligned}
$$




$$
\begin{aligned}
& =\int_{-\infty}^{\infty} P\left(\bigcup_{0 \leq t}\left\{W_{t+v}-W_{v} \geq a t+a v+\tilde{b}-y\right\}\right) P\left(W_{v} \in d y\right) \\
& =\int_{-\infty}^{\infty} P\left(\bigcup_{0 \leq t}\left\{W_{t} \geq a t+a v+\tilde{b}-y\right\}\right) P\left(W_{v} \in d y\right) .
\end{aligned}
$$

Assuming that $a>0$ and applying the case i) of Proposition 3.1 in (15) we get

$$
\begin{aligned}
& P\left(\bigcup_{v \leq t}\left\{W_{t} \geq a t+\tilde{b}\right\}\right) \\
& \quad=\int_{-\infty}^{a v+\tilde{b}} \exp (-2\{a v+\tilde{b}-y\} a) \phi(0, v) d y+\int_{a v+\tilde{b}}^{\infty} \phi(0, v) d y
\end{aligned}
$$

Using the properties of the normal distribution and the following integration formula

$$
\int_{-\infty}^{k} \exp \left(-\left\{a x^{2}+b x\right\}\right) d x=\exp \left(\frac{b^{2}}{4 a}\right) \sqrt{\frac{\pi}{a}} \Phi\left(\frac{2 k a+b}{\sqrt{2 a}}\right)
$$

in expression (16), we get

$$
\begin{aligned}
P\left(\bigcup_{v \leq t}\left\{W_{t} \geq a t+\tilde{b}\right\}\right)= & \exp (-2 a \tilde{b}) \Phi\left(\frac{\tilde{b}}{\sqrt{v}}-a \sqrt{v}\right) \\
& +1-\Phi\left(\frac{\tilde{b}}{\sqrt{v}}+a \sqrt{v}\right) .
\end{aligned}
$$

Finally the result is obtained if we replace $\tilde{b}=\frac{a}{T}-b-\frac{x}{T}$ in the above equality. This ends the proof.

Remark 3.3. If $r \rightarrow T^{-}$in (12), we readily obtain the result (11).

The second result is the following.

Theorem 3.4. Let be $T<r$. If $a>0$ and $x<a-b T$ then

$$
\begin{aligned}
P\left(\bigcup_{0 \leq t \leq r}\left\{W_{t} \geq a-b t\right\} \mid W_{T}=x\right) \\
=\exp (-2 a(a-x-b T) / T)\left[\Phi\left(\frac{a-x-b T}{\sqrt{r-T}}-b \sqrt{r-T}\right)\right. \\
\left.\quad-\exp (2(a-x-b T) b) \Phi\left(-b \sqrt{r-T}-\frac{a-x-b T}{\sqrt{r-T}}\right)\right]+1-\Phi\left(\frac{a-x-b T}{\sqrt{r-T}}-b \sqrt{r-T}\right) \\
\quad+\exp (2(a-x-b T) b) \Phi\left(-b \sqrt{r-T}-\frac{a-x-b T}{\sqrt{r-T}}\right) .
\end{aligned}
$$

If $x \geq a-b T$ then the probability is 1.

Proof. First, we observe that

$$
\begin{aligned}
& P\left(\bigcup_{0 \leq t \leq r}\left\{W_{t} \geq a-b t\right\} \mid W_{T}=x\right) \\
&= P\left(\bigcup_{0 \leq t \leq T}\left\{W_{t} \geq a-b t\right\} \mid W_{T}=x\right)+P\left(\bigcup_{T \leq t \leq r}\left\{W_{t} \geq a-b t\right\} \mid W_{T}=x\right) \\
&-P\left(\bigcup_{0 \leq t \leq T}\left\{W_{t} \geq a-b t\right\}, \bigcup_{T \leq t \leq r}\left\{W_{t} \geq a-b t\right\} \mid W_{T}=x\right)
\end{aligned}
$$


Now, we use the Markov property and invariance properties of Brownian motion to calculate the joint probability in the last term of (18).

$$
\begin{aligned}
& P\left(\bigcup_{0 \leq t \leq T}\left\{W_{t} \geq a-b t\right\}, \bigcup_{T \leq t \leq r}\left\{W_{t} \geq a-b t\right\} \mid W_{T}=x\right) \\
& =P\left(\bigcup_{0 \leq t \leq T}\left\{W_{t} \geq a-b t\right\} \mid W_{T}=x\right) P\left(\bigcup_{T \leq t \leq r}\left\{W_{t} \geq a-b t\right\} \mid W_{T}=x\right) \\
& =P\left(\bigcup_{0 \leq t \leq T}\left\{W_{t} \geq a-b t\right\} \mid W_{T}=x\right) P\left(\bigcup_{0 \leq t \leq r-T}\left\{W_{t} \geq a-b T-x-b t\right\}\right) \\
& =P\left(\bigcup_{0 \leq t \leq T}\left\{W_{t} \geq a-b t\right\} \mid W_{T}=x\right) \\
& \left.\quad+\exp (2(a-x-b T) b) \Phi\left(-b \sqrt{r-T}-\frac{a-x-b T}{\sqrt{r-T}}\right)\right],
\end{aligned}
$$

where the last equality in (19) is consequence of case ii) of Proposition 3.1. Therefore, from (18) and (19) we get

$$
\begin{aligned}
& P\left(\bigcup_{0 \leq t \leq r}\left\{W_{t} \geq a-b t\right\} \mid W_{T}=x\right) \\
& \quad=P\left(\bigcup_{0 \leq t \leq T}\left\{W_{t} \geq a-b t\right\} \mid W_{T}=x\right)\left[\Phi\left(\frac{a-x-b T}{\sqrt{r-T}}-b \sqrt{r-T}\right)\right. \\
& \left.\quad-\exp (2(a-x-b T) b) \Phi\left(-b \sqrt{r-T}-\frac{a-x-b T}{\sqrt{r-T}}\right)\right] \\
& \quad+1-\Phi\left(\frac{a-x-b T}{\sqrt{r-T}}-b \sqrt{r-T}\right)+\exp (2(a-x-b T) b) \Phi\left(-b \sqrt{r-T}-\frac{a-x-b T}{\sqrt{r-T}}\right)
\end{aligned}
$$

Finally, we use equality (11) to obtain

$$
\begin{aligned}
& P\left(\bigcup_{0 \leq t \leq r}\left\{W_{t} \geq a-b t\right\} \mid W_{T}=x\right) \\
& \quad=\exp (-2 a(a-x-b T) / T)\left[\Phi\left(\frac{a-x-b T}{\sqrt{r-T}}-b \sqrt{r-T}\right)\right. \\
& \left.\quad-\exp (2(a-x-b T) b) \Phi\left(-b \sqrt{r-T}-\frac{a-x-b T}{\sqrt{r-T}}\right)\right] \\
& \quad+1-\Phi\left(\frac{a-x-b T}{\sqrt{r-T}}-b \sqrt{r-T}\right)+\exp (2(a-x-b T) b) \Phi\left(-b \sqrt{r-T}-\frac{a-x-b T}{\sqrt{r-T}}\right) .
\end{aligned}
$$

For the case $x \geq a-b T$ it is satisfied that

$$
\begin{gathered}
P\left(\bigcup_{0 \leq t \leq T}\left\{W_{t} \geq a-b t\right\}, \bigcup_{T \leq t \leq r}\left\{W_{t} \geq a-b t\right\} \mid W_{T}=x\right) \\
=P\left(\bigcup_{0 \leq t \leq T}\left\{W_{t} \geq a-b t\right\} \mid W_{T}=x\right)
\end{gathered}
$$

and in consequence we get that the probability (18) is equal to 1 . This finishes the proof.

Remark 3.5. If $r \rightarrow T^{+}$in expression (17), we readily obtain the result (11). 
Remark 3.6. Theorem 3.4 is equivalent to a particular case of the result presented in [1, Theorem 2.4].

Finally, we are ready to prove the main result of the paper. To simplify the notation we define for $x \in \mathbb{R}$

$$
R(t, x)=\frac{1}{c_{2}(p-1)(g(x))^{p-1}}-\frac{c_{1}}{c_{2}} t .
$$

Proof of the Theorem 1.2. Let $r \geq 0$. Then

$$
\begin{aligned}
P(\tau \leq r) & =\int_{-\infty}^{\infty} P\left(\inf _{0 \leq t}\left\{W_{t} \geq R\left(t, W_{T}\right)\right\} \leq r \mid W_{T}=x\right) P\left(W_{T} \in d x\right) \\
& =\int_{-\infty}^{\infty} P\left(\inf _{0 \leq t}\left\{W_{t} \geq R(t, x)\right\} \leq r \mid W_{T}=x\right) P\left(W_{T} \in d x\right) \\
& =\int_{-\infty}^{\infty} P\left(\bigcup_{t \in[0, r]}\left\{W_{t} \geq R(t, x)\right\} \mid W_{T}=x\right) P\left(W_{T} \in d x\right)
\end{aligned}
$$

The second equality in (20) is consequence of a well-known result, see [33, Property CE10, pp. 462].

First, we suppose that $r<T$. From case b) of Theorem 3.2 we have

$$
\begin{aligned}
& P\left(\bigcup_{t \in[0, r]}\left\{W_{t} \geq R(t, x)\right\} \mid W_{T}=x\right) \\
& =1-\Phi\left(\frac{R(T, x)-x}{T \sqrt{v}}+\frac{\sqrt{v}}{c_{2}(p-1)(g(x))^{p-1}}\right) \\
& \quad+\exp \left(\frac{-2(R(T, x)-x)}{T c_{2}(p-1)(g(x))^{p-1}}\right) \Phi\left(\frac{R(T, x)-x}{T \sqrt{v}}-\frac{\sqrt{v}}{c_{2}(p-1)(g(x))^{p-1}}\right) .
\end{aligned}
$$

In other hand, the random variable $W_{T} \sim \phi(0, T)$ and note that $a(x)=R(T, x)-x$. Therefore replacing equation (21) in the last equality in (20) we get the case i), i.e.,

$$
\begin{aligned}
P & (\tau \leq r) \\
& =1-\int_{-\infty}^{\infty} \Phi\left(\frac{a(x)}{T \sqrt{v}}+\frac{\sqrt{v}}{c_{2}(p-1)(g(x))^{p-1}}\right) \phi(0, T) d x \\
& +\int_{-\infty}^{\infty} \exp \left(\frac{-2 a(x)}{T c_{2}(p-1)(g(x))^{p-1}}\right) \Phi\left(\frac{a(x)}{T \sqrt{v}}-\frac{\sqrt{v}}{c_{2}(p-1)(g(x))^{p-1}}\right) \phi(0, T) d x .
\end{aligned}
$$

Now, we assume that $T<r$. From Theorem 3.4, if $x<R(T, x)$, we obtain

$$
\begin{aligned}
& P\left(\bigcup_{t \in[0, r]}\left\{W_{t} \geq R(t, x)\right\} \mid W_{T}=x\right) \\
& =\exp \left(\frac{-2(R(T, x)-x)}{T c_{2}(p-1)(g(x))^{p-1}}\right)\left[\Phi\left(\frac{R(T, x)-x}{\sqrt{r-T}}-\frac{c_{1} \sqrt{r-T}}{c_{2}}\right)\right. \\
& \left.\quad-\exp \left(\frac{2(R(T, x)-x) c_{1}}{c_{2}}\right) \Phi\left(\frac{-R(T, x)+x}{\sqrt{r-T}}-\frac{c_{1} \sqrt{r-T}}{c_{2}}\right)\right] \\
& +1-\Phi\left(\frac{R(T, x)-x}{\sqrt{r-T}}-\frac{c_{1} \sqrt{r-T}}{c_{2}}\right) \\
& +\exp \left(\frac{2(R(T, x)-x) c_{1}}{c_{2}}\right) \Phi\left(\frac{-R(T, x)+x}{\sqrt{r-T}}-\frac{c_{1} \sqrt{r-T}}{c_{2}}\right)
\end{aligned}
$$

and

$$
P\left(\bigcup_{t \in[0, r]}\left\{W_{t} \geq R(t, x)\right\} \mid W_{T}=x\right)=1
$$


if $x \geq R(T, x)$. Therefore we separate the integral in expression (20)

$$
\begin{aligned}
P(\tau \leq r)= & \\
= & \int_{-\infty}^{\infty} P\left(\bigcup_{t \in[0, r]}\left\{W_{t} \geq R(t, x)\right\} \mid W_{T}=x\right) I_{\{x<R(T, x)\}} \phi(0, T) d x \\
& +\int_{-\infty}^{\infty} I_{\{x \geq R(T, x)\}} \phi(0, T) d x,
\end{aligned}
$$

and replacing expressions (22) and (23) in the above equality to get

$$
\begin{aligned}
& P(\tau \leq r) \\
& =\int_{-\infty}^{\infty} \exp \left(\frac{-2 a(x)}{T c_{2}(p-1)(g(x))^{p-1}}\right) \Phi\left(\frac{a(x)}{\sqrt{r-T}}-\frac{c_{1} \sqrt{r-T}}{c_{2}}\right) I_{\{x<R(T, x)\}} \phi(0, T) d x \\
& \quad-\int_{-\infty}^{\infty} \exp \left(\frac{-2 a(x)}{T c_{2}(p-1)(g(x))^{p-1}}+\frac{2 a(x) c_{1}}{c_{2}}\right) \\
& \quad \times \Phi\left(\frac{-a(x)}{\sqrt{r-T}}-\frac{c_{1} \sqrt{r-T}}{c_{2}}\right) I_{\{x<R(T, x)\}} \phi(0, T) d x \\
& +1-\int_{-\infty}^{\infty} \Phi\left(\frac{a(x)}{\sqrt{r-T}}-\frac{c_{1} \sqrt{r-T}}{c_{2}}\right) I_{\{x<R(T, x)\}} \phi(0, T) d x \\
& \quad+\int_{-\infty}^{\infty} \exp \left(\frac{2 a(x) c_{1}}{c_{2}}\right) \Phi\left(\frac{-a(x)}{\sqrt{r-T}}-\frac{c_{1} \sqrt{r-T}}{c_{2}}\right) I_{\{x<R(T, x)\}} \phi(0, T) d x,
\end{aligned}
$$

where the case iii) is obtained using that $a(x)=R(T, x)-x$. Finally, for the case ii), we compute the limit when $r \rightarrow T$. Dominated Convergence Theorem and the continuity of the standard normal distribution imply

$$
\begin{aligned}
\lim _{r \rightarrow T^{-}}\left\{1-\int_{-\infty}^{\infty} \Phi\left(\frac{a(x)}{T \sqrt{v}}+\frac{\sqrt{v}}{c_{2}(p-1)(g(x))^{p-1}}\right) \phi(0, T) d x\right. \\
\left.+\int_{-\infty}^{\infty} \exp \left(\frac{-2 a(x)}{T c_{2}(p-1)(g(x))^{p-1}}\right) \Phi\left(\frac{a(x)}{T \sqrt{v}}-\frac{\sqrt{v}}{c_{2}(p-1)(g(x))^{p-1}}\right) \phi(0, T) d x\right\} \\
=1-\int_{-\infty}^{\infty} \Phi(\infty) I_{\{a(x)>0\}} \phi(0, T) d x \\
+\int_{-\infty}^{\infty} \exp \left(\frac{-2 a(x)}{T c_{2}(p-1)(g(x))^{p-1}}\right) \Phi(\infty) I_{\{a(x)>0\}} \phi(0, T) d x \\
=\lim _{r \rightarrow T}\left\{\int_{-\infty}^{\infty} \exp \left(\frac{-2 a(x)}{T c_{2}(p-1)(g(x))^{p-1}}\right) \Phi\left(\frac{a(x)}{\sqrt{r-T}}-\frac{c_{1} \sqrt{r-T}}{c_{2}}\right) I_{\{a(x)>0\}} \phi(0, T) d x\right. \\
\quad-\int_{-\infty}^{\infty} \exp \left(\frac{-2 a(x)}{T c_{2}(p-1)(g(x))^{p-1}}+\frac{2 a(x) c_{1}}{c_{2}}\right) \\
\quad+1-\int_{-\infty}^{\infty} \Phi\left(\frac{a(x)}{\sqrt{r-T}}-\frac{c_{1} \sqrt{r-T}}{c_{2}}\right) I_{\{a(x)>0\}} \phi(0, T) d x \\
\left.\quad+\int_{-\infty}^{\infty} \exp \left(\frac{2 a(x) c_{1}}{c_{2}}\right) \Phi\left(\frac{-a(x)}{\sqrt{r-T}}-\frac{c_{1} \sqrt{r-T}}{c_{2}}\right) I_{\{a(x)>0\}} \phi(0, T) d x\right\} .
\end{aligned}
$$

The above concludes the proof.

Remark 3.7. Using the case a) of Theorem 3.2 in equation (20) we also get the probability ii) in Theorem 1.2. 


\section{Further remarks and open problems}

We devote this section to present some conclusion remarks and further discussion.

- In this paper, we have considered a stochastic model for fatigue in which the initial condition is given by a non-adapted random variable to the original filtration. Although the tools of anticipating calculus have been extensively used in finance applications (see, e.g., [15], [17], [19], [21]), to the best of the author knowledge, it has been rarely studied outside this context. Nevertheless, works as [25] and references within show that studying the anticipating case in certain experiments of physics may yield results that approximate better the actual phenomena. Here, we have presented only a first attempt in incorporating anticipating tools to the study of fatigue models. In practice, this would represent that the dominant crack is being externally affected at a certain time $T>0$ and the knowledge of this event is known and taken into account from the very beginning at $t=0$.

- In this paper the distribution function of $\tau$, the time of the ultimate damage, is provided. Accordingly, this work can be considered as an interesting application of the explosion phenomenon in anticipating SDE which is currently an area of very fruitful research. In fact, to the best of our knowledge, there exists only few works where the law of the blow up time of non anticipating SDE is studied (the interested reader can consult, for instance [11], [16], [23]). In the case of anticipating SDE, the results are far from being completed and this work represent a further step in this direction.

- For models with explosion is relevant to have numerical schemes that reproduce this behavior. For the case of non-anticipating SDE with coefficients without dependence of the time (where the model (1) is included) in [9] the authors use the Euler-Maruyama scheme to simulate solutions of SDE with explosion. Indeed, they select the step time of the method base on the Osgood test for explosion ([22], [30]), i.e.,

$$
T_{k}=\frac{h}{b\left(X_{k}\right)} \quad \text { for } \quad h>0,
$$

in equation

$$
X_{k+1}=X_{k}+T_{k} b\left(X_{k}\right)+\sigma\left(X_{k}\right) \Delta W_{k},
$$

where $b, \sigma$ are the drift and diffusion coefficients respectively. However, as far as we know, the techniques and tools used to work with no-adapted stochastic integrals cannot be carried out easily to the Euler schemes, therefore, there are only few works related on (see for example [2], [39]), in consequence, this seems as an interesting and challenging problem from the mathematical point of view.

\section{References}

[1] M. Abundo, Some conditional crossing results of Brownian motion over a piecewise-linear boundary, Statist. Probab. Lett., 58 (2002), pp. 131-145.

[2] H. Ahn And A. Kohatsu-Higa, The Euler scheme for anticipating stochastic differential equations, Stochastics Stochastics Rep., 54 (1995), pp. 247-269.

[3] A. Aksamit and M. Jeanblanc, Enlargement of filtration with finance in view, SpringerBriefs in Quantitative Finance, Springer, Cham, 2017.

[4] L. ARnold, Stochastic differential equations: theory and applications, Wiley-Interscience [John Wiley \& Sons], New York-London-Sydney, 1974. Translated from the German.

[5] P. Augustin, Simulation of fatigue crack growth in integrally stiffened panels under the constant amplitude and spectrum loadin, Fatigue of Aircraft Structures, 1 (2009), pp. 5-19.

[6] L. Beghin And E. Orsingher, On the maximum of the generalized Brownian bridge, Liet. Mat. Rink., 39 (1999), pp. 200-213.

[7] A. N. Borodin And P. SAlminen, Handbook of Brownian motion-facts and formulae, Probability and its Applications, Birkhäuser Verlag, Basel, second ed., 2002. 
[8] C. C. Chamis, Damage tolerance and reliability of turbine engine components, NASA Glenn Research Center, (1999).

[9] J. Dávila, J. F. Bonder, J. D. Rossi, P. Groisman, and M. Sued, Numerical analysis of stochastic differential equations with explosions, Stoch. Anal. Appl., 23 (2005), pp. 809825 .

[10] J. L. Doob, Heuristic approach to the Kolmogorov-Smirnov theorems, Ann. Math. Statistics, 20 (1949), pp. 393-403.

[11] W. Feller, Diffusion processes in one dimension, Trans. Amer. Math. Soc., 77 (1954), pp. 1-31.

[12] A. C. Ford, H. Gramling, C. L. Samuel, J. V. Sov, A. Srinivasan, and L. A. Prui, Micromechanisms of fatigue crack growth in polycarbonate polyurethane:time dependent and hydration effects, Journal of the Mechanical Behavior of Biomedical Materials, 79 (2018), pp. 324-331.

[13] N. E. Frost And D. S. Dugdale, The propagation of fatigue cracks in sheet specimens, vol. 6, J. Mech. Phys. Solids, 1958.

[14] K. Itô, Stochastic integral, Proc. Imp. Acad. Tokyo, 20 (1944), pp. 519-524.

[15] H. JAFARI AND G. RAHIMI, Small-time asymptotics in geometric Asian options for a stochastic volatility jump-diffusion model, Int. J. Theor. Appl. Finance, 22 (2019), pp. 1950005, 19.

[16] I. Karatzas And J. RuF, Distribution of the time to explosion for one-dimensional diffusions, Probab. Theory Related Fields, 164 (2016), pp. 1027-1069.

[17] A. Kohatsu-Higa, Models for insider trading with finite utility, in Paris-Princeton Lectures on Mathematical Finance 2004, vol. 1919 of Lecture Notes in Math., Springer, Berlin, 2007, pp. 103-171.

[18] A. Kohatsu-Higa AND J. A. LEÓN, Anticipating stochastic differential equations of Stratonovich type, Appl. Math. Optim., 36 (1997), pp. 263-289.

[19] A. Kohatsu-Higa And A. Sulem, Utility maximization in an insider influenced market, Math. Finance, 16 (2006), pp. 153-179.

[20] J. M. LEE, Introduction to smooth manifolds, vol. 218 of Graduate Texts in Mathematics, Springer, New York, second ed., 2013.

[21] J. A. León, R. NAvarro, and D. Nualart, An anticipating calculus approach to the utility maximization of an insider, vol. 13, 2003, pp. 171-185. Conference on Applications of Malliavin Calculus in Finance (Rocquencourt, 2001).

[22] J. A. León AND L. Peralta, Some Feller and Osgood type criteria for semilinear stochastic differential equations, Stoch. Dyn., 17 (2017), pp. 1750011, 19.

[23] J. A. León, L. Peralta Hernández, and J. Villa-Morales, On the distribution of explosion time of stochastic differential equations, Bol. Soc. Mat. Mexicana (3), 19 (2013), pp. $125-138$.

[24] K. Y. Lin And A. V. STYUAR, Probabilistic approach to damage tolerance design of aircraft composite structures, J Aircr, 44 (2007), pp. 1309-1317.

[25] R. Mannella and P. V. E. Mcclintock, Ito versus stratonovich: 30 years later, Fluctuation and Noise Letters, 11 (2012), pp. 1240010-1-1240010-10.

[26] H. P. McKean, Stochastic integrals, AMS Chelsea Publishing, Providence, RI, 2005. Reprint of the 1969 edition, with errata.

[27] L. Molent, M. McDonald, S. Barter, And R. Jones, Evaluation of spectrum fatigue crack growth using variable amplitude data, International Journal of Fatigue, 30 (2008), pp. 119-137.

[28] N. A. Navarro, Dos modelos estocásticos para transacciones con información privilegiada en mercados financieros y dependencia con memoria larga en tiempos de ocupación, Ph.D. Thesis, Centro de investigación CINVESTAV IPN, (2004). 
[29] D. Nualart, Noncausal stochastic integrals and calculus, in Stochastic analysis and related topics (Silivri, 1986), vol. 1316 of Lecture Notes in Math., Springer, Berlin, 1988, pp. 80129 .

[30] W. F. OsGood, Beweis der Existenz einer Lösung der Differentialgleichung $\frac{d y}{d x}=f(x, y)$ ohne Hinzunahme der Cauchy-Lipschitz'schen Bedingung, Monatsh. Math. Phys., 9 (1898), pp. 331-345.

[31] E. Pardoux, Applications of anticipating stochastic calculus to stochastic differential equations, in Stochastic analysis and related topics, II (Silivri, 1988), vol. 1444 of Lecture Notes in Math., Springer, Berlin, 1990, pp. 63-105.

[32] P. Paris And F. ERdogan, A critical analysis of crack propagation laws, J. Fluids Eng, 85 (1963), pp. 528-533.

[33] P. E. Pfeiffer, Probability for applications, Springer Texts in Statistics, Springer-Verlag, New York, 1990.

[34] D. Revuz And M. Yor, Continuous martingales and Brownian motion, vol. 293 of Grundlehren der Mathematischen Wissenschaften [Fundamental Principles of Mathematical Sciences], Springer-Verlag, Berlin, third ed., 1999.

[35] F. Russo And P. VAllois, Forward, backward and symmetric stochastic integration, Probab. Theory Related Fields, 97 (1993), pp. 403-421.

[36] T. H. Scheike, A boundary-crossing result for Brownian motion, J. Appl. Probab., 29 (1992), pp. 448-453.

[37] K. Sobczyк, Modelling of random fatigue crack growth, Engineering Fracture Mechanics, 24 (1986), pp. 609-623.

[38] K. Sobczyk and B. J. Spencer., Random fatigue: From data to theory, Academic Press Inc., Boston, MA., 1992.

[39] S. TORRES AND C. A. Tudor, The Euler scheme for a class of anticipating stochastic differential equations, Random Oper. Stochastic Equations, 12 (2004), pp. 211-224.

[40] R. S. VALLURI, Some recent developments at GALCIT concerning a theory of metal fatigue, vol. 11, Acta Metall, 1963. 